\title{
Knowns and Unknowns of Influenza B Viruses
}

2

3 Marios Koutsakos ${ }^{1}$, Thi HO Nguyen ${ }^{1}$, Wendy S Barclay ${ }^{2}$ and Katherine

4 Kedzierska ${ }^{1 *}$

5

6

7 'Department of Microbiology and Immunology, University of Melbourne, at the

8 Peter Doherty Institute for Infection and Immunity, Parkville VIC 3010,

9 Australia; ${ }^{2}$ Section of Virology, Faculty of Medicine, Wright Fleming Institute,

10 Imperial College London, Norfolk Place, London W2 1PG, UK

13 Running title: Flu B virology and immunology

14 Keywords: influenza B, virology, epidemiology and immunology

$16{ }^{*}$ Correspondence should be addressed to:

17 Katherine Kedzierska, PhD

18 Department of Microbiology and Immunology

19 University of Melbourne

20 At the Peter Doherty Institute for Infection and Immunity

21 Vic 3010, Australia

22 Ph: +61 0383447962

23 Fax: +6103 93471540

24 kkedz@unimelb.edu.au 
28 Influenza B (FluB) viruses circulate annually along with influenza A (FluA)

29 strains during seasonal epidemics. FluB can dominate influenza seasons and

30 cause severe disease, particularly in children and adolescents. Research has

31 revealed interesting aspects of FluB and highlighted the importance of these

32 viruses in clinical settings. Yet, many important questions remain

33 unanswered. In this review, the clinical relevance of FluB is emphasized,

34 unique features are highlighted and gaps in knowledge pinpointed. Multiple

35 aspects of FluB epidemiology, evolution, virology and immunology are

36 discussed. Future research into FluB is needed to understand how we can

37 prevent severe disease in high-risk groups, especially children and elderly. 
In 1940, an outbreak of acute respiratory illness in children with clinical presentations identical to the contemporary influenza A epidemics led to the isolation of a new virus that was serologically distinct from previous influenza A isolates. This novel influenza virus was named Influenza B [1]. It is now well established that influenza A (FluA) and Influenza B (FluB) viruses co-circulate annually within the human population and cause respiratory illness with varying levels of severity. Unlike FluA, FluB does not have an established animal reservoir and thus does not pose the same pandemic risk as FluA [2]. Consequently, FluB has received considerably less attention. Nonetheless, FluB presents a substantial challenge during annual epidemics. Although current knowledge of FluB viruses highlights their unique features, a plethora of questions on the virology, immunology, epidemiology and evolution of FluB remains to be elucidated.

FluB viruses belong to the family of Orthomyxoviruses and have a segmented negative-sense single-stranded RNA genome. FluB has 8 53 segments and encodes for 3 polymerase proteins (PA, PB1 and PB2), the nucleoprotein (NP), the non-structural protein (NS1), the nuclear export protein (NEP or sometimes called BNS2), the matrix protein (BM1), the BM2 ion channel and three surface glycoproteins (HA, NA and NB) [3] (Fig 1A-B).

57 FluA and FluB proteins generally show substantial differences in length, 58 amino acid composition and function [2]. FluB viruses are currently divided 59 into two genetically and antigenically distinct lineages, B/Victoria/2/1987-like 60 and B/Yamagata/16/1988-like viruses [4-6]. 
FluB viruses from one or both lineages co-circulate annually with the seasonal $\mathrm{H} 1 \mathrm{~N} 1$ and $\mathrm{H} 3 \mathrm{~N} 2$, with FluB being the main cause of the seasonal epidemic every 2-4 years $[7,8]$. Multiple lines of evidence suggest that FluB can cause substantial disease [9-11]. In an analysis of influenza-related mortality between 1976 and 1999 in the US, highest morbidity was associated with $\mathrm{H} 3 \mathrm{~N} 2$, followed by FluB and then by $\mathrm{H} 1 \mathrm{~N} 1$ [12]. This highlights that FluB infections can cause a significant degree of morbidity, although the analysis was conducted in 2009 and the above hierarchy might have changed with the establishment of the H1N1pdm strain in seasonal epidemics. Regardless, various reports highlight a strong presence of FluB in seasonal epidemics across the world. An epidemiological analysis of influenza-attributable mortality in the US between 1997 and 2009, reported that $29 \%$ of total influenza associated deaths were attributable to a FluB infection. Importantly, in 4 out of 12 seasons FluB infection was the predominant cause of seasonal influenza associated mortality [13]. In the 2008 season in Australia, 54\% of influenza notifications were due to FluB infections, and the majority of these infections were in children [14]. In England, in the first half of the 2012/2013 season, FluB was the predominant cause of influenza infections, with the highest activity occurring in children aged 5-14 [15]. Additionally, in Thailand between 2010 and 2014 the majority of FluB infections occurred in children aged 5-19 [16]. These are only a few studies indicating that FluB can cause severe disease, especially in the young. The factors determining the predominance of FluB over FluA in a given season are largely unknown, but most likely depend on specific viral characteristics and interactions from all

87 three circulating viruses. 

discharge and fever [17]. Overall, disease caused by FluB is indistinguishable from that of FluA [18], although complications can differ. Complications of

91 FluB can include neurological and muscular manifestations [19,20], 92 cardiologic complications [21-23] and secondary bacterial infections 93 [22,24,25]. Paddock et al (2012) observed that secondary bacterial 94 pneumonia was more frequently observed in adult fatal cases of FluB than 95 pediatric fatal cases, and the reverse was observed for cardiac complications. 96 The same study also determined that in fatal cases of influenza, the time from 97 disease onset to death was shorter for FluB than that determined for the 98 1918, 1957, 1968, 2009 pandemic FluA or seasonal H3N2 [22]. It is however 99 important to assess the validity of this observation in other FluB cohorts. It 100 also appears that lineage does not impact disease outcome [26]. As 101 mentioned above, although FluB can infect adults and the elderly, disease 102 manifestations are more prevalent in children [27-29]. Moreover, FluB viruses 103 of the Victoria lineage are more prevalent in younger populations than 104 Yamagata viruses $[6,30,31]$. It has been proposed that this could be explained 105 by higher expression of $\alpha-2,3$ linked sialic acid glycans in children than in 106 adults and the differential $\alpha-2,3$ and $\alpha-2,6$ sialic acid glycan binding 107 preferences of viruses from the two lineages [6,32]. Overall, FluB can cause 108 significant disease, particularly in children. The viral and host determinants of 109 FluB pathogenicity and the reasons of increased susceptibility of young 110 children remain to be elucidated.

\section{Evolution and Phylodynamics of FluB}


In the early decades of the $20^{\text {th }}$ century FluB viruses belonged to a

114 single lineage. Two antigenically distinct lineages emerged prior to 1980 [4-6]

115 and now co-circulate globally, with one lineage usually predominating in any

116 given year, although equal circulation of the two lineages in certain areas has

117 been reported. For instance in the 2011-2012 season in Canada, $24 \%$ of all

118 influenza cases were caused by B/Victoria-like viruses and $27 \%$ were of

119 B/Yamagata-like origin [33]. In general, after the appearance of the two

120 lineages, Victoria-like viruses dominated in the late 1980s, while viruses of the

121 Yamagata lineage prevailed in the 1990s, with the exception of an outbreak of

122 Victoria-like viruses in Asia in 1996/1997. The Victoria lineage re-emerged

123 globally in 2001, and the two lineages have co-circulated ever since [34].

124 Interestingly, while new $\mathrm{H} 3 \mathrm{~N} 2$ viruses seem to originate from East and

125 Southeast Asia in every season, FluB strains can persist during multiple

126 seasons and re-seed subsequent epidemics [35,36]. This appears to be

127 linked to less frequent global movements of FluB compared to H3N2 FluA 128 [35].

129 Evolution of FluB involves a constant turnover of antigenically-distinct 130 viruses, in a process similar to the antigenic drift of FluA, as well as 131 reassortment. The evolution rate of FluB is slower than that of FluA, as

132 calculated by the rate of nucleotide substitutions for each segment. $\left(0.14 \times 10^{-}\right.$

$133^{3}-3.32 \times 10^{-3}$ substitutions/site/year for FluB, $2.68 \times 10^{-3}-12.5 \times 10^{-3}$ 134 substitutions/site/year for FluA H3N2) [34,37]. HA exhibits the highest rate of 135 adaptive evolution, suggesting a degree of positive selection, depicting the 136 effect of immune evolutionary pressure [5,34] Additionally, the NA and NS1 137 segments exhibit high levels of adaptive evolution, possibly due to immune 
138 pressure [34]. In addition to these changes, reassortment also contributes to

139 FluB genetic diversity. Reassortment can occur between viruses of the same

140 lineage or viruses of the two distinct lineages [5,6,34,38]. Inter-lineage

141 reassortment, in which Victoria viruses acquire genes from the Yamagata

142 lineage have been more frequent than the reverse [6]. Interestingly, different

143 segments can exhibit different patterns during reassortment events. Two

144 bioinformatics studies revealed that only the PB1, PB2 and HA segments

145 have maintained separate Victoria or Yamagata lineages. In contrast, the

146 NS1/NS2 segment of current strains is derived from the Victoria lineage, while

147 the rest of the segments (PA, NP, NA/NB, M1/BM2) come from the Yamagata

148 lineage, suggesting substantial exchange of genetic information between the

149 two lineages [6,38]. The observation that novel reassortant viruses can

150 dominate the viral population implies that reassortment can generate novel

151 strains with increased fitness.

152 Intriguingly, the two lineages exhibit different phylodynamics. Distinct 153 clades of Victoria viruses generally circulate for 1-3 years before being 154 replaced by new clades, while multiple clades of Yamagata viruses can co155 circulate for longer periods of time. Additionally, Victoria viruses exhibit great 156 genetic diversity between seasons and a stronger antigenic drift compared to 157 Yamagata viruses. Furthermore, viruses of the Victoria lineage contain more 158 amino acid substitutions in the HA and these are located near the receptor 159 binding site of the HA, while changes in Yamagata viruses are less frequent 160 and tend to appear in sites more distant to the receptor binding site. Overall, 161 the Yamagata viruses are more conserved and show weaker antigenic drift 162 and selection than the faster evolving Victoria viruses [6]. 
164 between FluA and FluB. For instance, FluA (H1N1pdm09) infection can 165 prevent FluB infection within a short interval of time but the opposite is not 166 true, in the ferret model [39]. Additionally, analysis of FluA and FluB viruses

167 over 30 years revealed that in certain years, strong genetic bottlenecks have 168 been imposed on FluB viruses and these were associated with high 169 prevalence of FluA. Such bottlenecks have led to changes in the dominant 170 FluB antigenic cluster[34].

171 In summary, FluB evolution is a complex function of interactions 172 between FluB viruses of the different lineages, interactions between co173 circulating FluB and FluA viruses as well as the selective pressures from the 174 host's immune mechanisms.

Virology of FluB

\section{The Haemagglutinin (HA)}

The HA of FluB mediates attachment and entry of the virus into cells

180 via interaction with sialic acids. FluB HA binds to sialic acids with lower affinity 181 than FluA $[40,41]$. Also, the FluB HA can interact with both $\alpha-2,6-$ and $\alpha-2,3-$ 182 linked sialic acids. Despite their low sequence homology ( 20-30\%), the 183 overall structures of FluB and FluA HAs are similar. Similarly to FluA, the FluB 184 HA has an elongated membrane-proximal domain and a globular membrane185 distal domain. The functional form of the FluB HA is a homotrimer. The 186 Receptor Binding Site (RBS) of FluB HA has some significant differences to that of FluA HA. One important difference is located at amino acid position 95 
188 (FluB HA numbering), which in FluB is a phenylalanine, while in FluA is a 189 highly conserved tyrosine $[40,42]$. The Phe95 residue has been shown to be 190 involved in the ability of FluB HA to interact with both human-like and avian-

191 like receptor analogues and with the lower affinity of FluB HA. Replacement of 192 Phe95 with a tyrosine residue increased receptor binding to levels 193 comparable to FluA and reduced binding to avian-like receptor analogues 194 [43]. The role of the HA protein in determining pathogenicity of FluA is well 195 established [44], however this has not been studied for FluB.

The Neuraminidase (NA)

The overall structure of FluB NA is similar to that of FluA NA, despite only $30 \%$ sequence homology. The active form of the FluB NA is a tetramer and each monomer contains two glycosylation sites (Asn283 and Asn143) 201 and a calcium-ion binding site. Asn283 is specific for FluB, while Asn143 is 202 conserved among Influenza viruses [45]. Nineteen highly conserved residues 203 form the NA active site of influenza A and B viruses. Residues in the catalytic 204 site (R118, D151, R152, R224, E276, R292, R371, and Y406) directly interact 205 with sialic acids, while framework residues structurally support the enzymatic 206 active site (E119, R156, W178, S179, D198, I222, E227, H274, E277, N294, 207 and E425). Neuraminidase inhibitors (NAIs) licensed in the clinic for treatment 208 of influenza infection appear to be less effective against FluB [46].

\section{The NB Protein}

The NB protein is uniquely encoded by FluB in segment 6, overlapping 212 with the NA coding sequence and is not present in FluA. The start codon of 
213 NB is located 4 nucleotides upstream of the NA start codon [47]. NB is an

214 integral membrane glycoprotein of 100 amino acids that is incorporated into 215 the virion [48]. The N-terminus contains two N-glycosylation sites, which are 216 further modified by the addition of multiple $\mathrm{N}$-acetyl-glucosamine residues 217 [49]. Palmitoylation of C-terminal residues is important for trafficking of NB to 218 the cell surface [50]. NB is dispensable for virus rescue and in vitro replication 219 [51] (unpublished observations Elderfield, Koutsakos \& Barclay). Hatta and 220 Kawaoka observed attenuation in absence of NB in BALB/c mice [51], 221 however, this could be due to an altered NA activity as the mutations 222 introduced have been previously reported to affect the NA expression [47]. In 223 contrast, our data suggest that a NB-knockout virus with unaffected NA 224 activity replicates equivalently to the WT virus in C57B/6 mice. This mutant 225 virus showed no fitness cost in ferrets and also transmitted to a new host, 226 although overall transmission in that study was low (unpublished observations 227 Elderfield, Koutsakos \& Barclay). This apparent redundancy of NB is 228 surprising and could be due to a host-specific role of NB. Additionally, the in 229 vivo role of NB has only been assessed in the early stages of infection (up to 230 day 4). It is possible that NB plays a role in the later stages of FluB infection. In various experimental settings, NB has displayed the ability to 232 conduct ions. In Xenopus oocytes, NB could stimulate the conductance of $\mathrm{Cl}^{-}$ 233 ions [52], while in E.coli-derived membranes NB was permeable to protons 234 [53]. Additionally, purified protein and peptides corresponding to the 235 transmembrane region of NB incorporated into artificial lipid bilayers could 236 form cation-selective channels [54,55]. A review of these studies questioned 237 the observed ion channel activity due to the limitations of the experimental 
systems utilized, especially as previous observations showed that membrane

239 proteins with no known ion channel activity were able to form multimers and 240 conduct ions in similar experimental settings [56]. Subsequently, it was 241 reported that a single amino acid substitution (S20A) in the NB 242 transmembrane domain resulted in altered gating and proton permeability, 243 supporting the idea of a NB ion channel. However, unlike the AM2, BM2, and $244 \mathrm{CM} 2$ ion channels of FluA, FluB and Influenza C viruses, NB has no $\mathrm{pH}-$ 245 modulating activity in the trans-Golgi network (TGN), which the AM2, BM2 246 and CM2 ion channels do [57]. It is therefore unclear whether NB operates as 247 an ion channel. Overall, more than 30 years after the discovery of NB, its role 248 and function remain enigmatic. Understanding the role of NB could benefit the 249 design of novel live-attenuated vaccines [51].

\section{The Matrix Protein (BM1)}

Very little is known about the M1 protein of FluB, which is encoded in 253 segment 7. M1 plays an important role in FluB adaptation in mice and 254 attenuation of the master donor vaccine strain (B/Ann Arbor/1/66) of the Live 255 Attenuated Influenza Vaccine (LAIV) [58]. The functional role of M1 in the life 256 cycle of FluB is not entirely known. M1 contains a Nuclear Localization Signal 257 (NLS), two Nuclear Export Signals (NES) and can act as a shuttling protein 258 between the nucleus and the cytoplasm in infected cells. All of these features 259 were necessary for the function of $M 1$, as they were required for the 260 production of viable virus [59]. Thus, the exact role of BM1, and whether it is 261 of a similar function to that of FluA M1, remains to be determined. 


\section{The BM2 Ion Channel}

Like in FluA, segment 7 encodes for a second protein, namely BM2 [60]. The BM2 protein is a small hydrophobic, integral membrane protein [61] that is synthesized in the late stages of infection [62]. The protein is transported through the TGN to the cell surface [63] and is incorporated in the virions [62]. The $\mathrm{N}$-terminus of the protein appears to be important for its transport within infected cells [63]. An interaction of BM2 with M1 has been proposed [64], supporting previous observations that BM2 is involved in the incorporation of the vRNP in the virions [65]. Additionally, BM2 is necessary for the production of viable virus [65-67] and decreased incorporation of BM2 in virions reduces infectivity, indicating a role for BM2 similar to that of AM2 [66]. Indeed, BM2 contains a HXXXW motif like AM2 and thus can conduct ions. This activity is similar to the one of AM2 in acidifying the endosome and modulating $\mathrm{pH}$ in the Golgi [68]. The crystal structure of BM2 reveals similarities to AM2 but also unique features. BM2 forms coiled-coil tetramers, with polar residues lining the pore that is formed, a feature important for the inactivity of adamantanes against BM2 [69]. Zhang et al, recently suggested a novel role for BM2 in inhibiting p53-mediated transcription and apoptosis [70].

A unique feature of BM2 is its expression mechanism. The BM2 coding sequence lies directly downstream of the M1 coding sequence and the two are translated from the same mRNA molecule. The translation termination codon of M1 overlaps with the start codon of BM2 in the following pentanucleotide sequence: UAAUG (M1 stop-codon underlined, BM2 start codon in bold) [71]. The 45 nucleotides in the M1 coding sequence upstream of penta- 
288 nucleotide appear essential for BM2 expression [72]. Additionally, the 289 sequence upstream of the penta-nucleotide exhibits complementarity to the $29018 S$ component of the small ribosomal subunit [73]. An interaction between 291 the mRNA and the rRNA as well as the involvement of the translation initiation 292 factor elF3 are important for BM2 expression [74]. This termination:reinitiation 293 mechanism was the first one of its kind when initially identified, but since then 294 similar mechanisms have been identified in other viruses, like caliciviruses 295 [73].

\section{The Nucleocapsid Protein (NP)}

The NP of FluB (BNP) is encoded by segment 5 . It differs greatly from 299 the NP of FluA (ANP). The most striking differences are the lack of the two 300 NLSs of ANP and the extended N-terminus of the protein in FluB. Specifically, 301 BNP contains 50 amino acids in its N-terminus that are not found in ANP [75].

302 Structural studies revealed that BNP consists of a head, a body and a 303 flexible tail loop. The groove between the head and the body contains an 304 extended charged loop (residues 125-149), within which there are two lysine 305 clusters critical for RNA binding. The flexible tail loop of BNP enables the 306 assembly of homo-oligomers. Comparisons with the structure of ANP 307 revealed an interesting similarity. In both proteins, a highly conserved salt 308 bridge (R472-E395 in BNP, R416-E339 in ANP) seems to be involved in the 309 stability of the loop and thus the assembly of homo-oligomers [76]. 310 Pharmacological inhibition of this salt bridge in ANP can inhibit FluA [77], and 311 thus might be a possible drug target for FluB. 
313 considerably different from ANP. Wanitchang et al showed that the N-

314 terminus of BNP is required for nuclear localization and suggested that the

$315 \mathrm{~K}_{44} \mathrm{RTR}_{47}$ motif in BNP might be acting as a NLS [78]. Reverse genetics were

316 later employed to generate FluB lacking the $\mathrm{N}$-terminus of $\mathrm{BNP}$, in order to

317 investigate the role of the $\mathrm{N}$-terminus of BNP in the context of infection and

318 viral replication. Different mutations in the N-terminus affected the ability to

319 rescue virus or viral replication and transcription. Moreover, the entire $\mathrm{N}$ -

320 terminus appears to be essential for optimal localization in the nucleus during

321 infection and residues 51-81 were suggested to contain a bipartite NLS [79].

322 The NP protein of FluA is known to have multiple functions that are crucial for

323 effective virus replication, however, there are no similar studies for BNP.

324 Current knowledge suggests that BNP has features similar to ANP but also 325 possesses some unique domains, suggesting that at least some functions of 326 the two proteins are unique.

The Polymerases (PA, PB1 \& PB2)

329 Although there is a vast amount of knowledge about the polymerases 330 of FluA, minimal data exist on the polymerase proteins of FluB, with the 331 general view being that they work similarly to those of FluA. However it is 332 pertinent that the FluB heterotrimeric polymerase complex structure was 333 solved before that of a human or avian FluA virus [80]. Indeed, similarities 334 exist, although recent studies suggest key differences. Localization studies 335 showed that all three FluB proteins have inherent nuclear import function and 336 encode NLSs. PB2 was found to predominantly localize in the nucleus, while 
PB1 and PA were localized both in the nucleus and the cytoplasm. Pairwise

338 interactions for all three proteins were also observed and localized in the 339 nucleus. These observations are similar to what has been observed for FluA 340 polymerase proteins [81].

A major difference between FluA and FluB polymerases is their capbinding mechanism. Specifically FluB polymerases can bind to and cleave

343 both $\mathrm{m}^{7} \mathrm{G}$-capped and unmethylated G-capped mRNAs. This is in contrast to

344 FluA polymerases that exclusively bind to and cleave $\mathrm{m}^{7} \mathrm{G}$-capped mRNA 345 [82]. Structural studies suggested that the overall structure and binding of the 346 polymerases to the vRNA is similar between FluA and FluB [80], but also 347 elucidate the details of the unique cap-binding mechanism of FluB [83]. The 348 polymerases of FluA have central roles in host-adaptation and pathogenicity $349[44,84]$. Conversely, the contributions of FluB polymerases to pathogenicity 350 and host-restriction are unknown.

\section{The Non-Structural 1 Protein (NS1)} The NS1 protein of FluB (NS1B) is a 281 amino acid long protein encoded by segment 8 . NS1B is important for virus replication as knock-out 355 viruses display impaired replicative capacity both in vitro [85] and in vivo [86]. 356 It consists of an N-terminus (residues 1-90), a C-terminus (residues 120-281) 357 and a linker domain (residues 91-119) [87]. NS1B localizes in the nucleus of 358 infected cells in the early stages of infection, but mostly in the cytoplasm in 359 the later stages. Residues $46-56$ in the N-terminus function as a monopartite

360 NLS and NS1B interacts with importin $\alpha 3$. Within the nucleus, NS1B localizes 361 in nuclear speckles containing splicing factor SC35 [88]. Although NS1B can 
bind RNA via its $\mathrm{N}$ terminus it cannot not inhibit export of host mRNA like

363 NS1A does, as it does not interact with CPSF30 via its C terminus [89]. NS1B can antagonize multiple aspects of the interferon antiviral

365 response $[85,90]$. Firstly, it can inhibit the IFN $\alpha \beta$ response by preventing 366 activation and nuclear translocation of IRF3. Particularly, FluB lacking NS1B 367 induces stronger IFN $\beta$ expression and activation of the IFN $\beta$ promoter than 368 WT FluB [85]. Additionally, ectopic overexpression of NS1B can inhibit IFN $\beta$ 369 promoter activity [90]. Interestingly, NS1B can complement the attenuated 370 growth of $\Delta$ NS1A FluA [90]. All these studies implicate NS1B as a potent 371 inhibitor of the IFN response. Both the $\mathrm{N}$ - and $\mathrm{C}$ - termini are involved in this 372 function. The N-terminus of NS1B sequesters dsRNA to prevent activation of 373 the antiviral PKR kinase, while the C-terminus is more involved in IFN $\beta$ 374 promoter inhibition [91], although either termini could independently prevent 375 activation of IRF-3 [90]. Interstingly, NS1B knock-out viruses are attenuated in 376 IFN-deficient Vero cells [85], suggesting that NS1B has additional effects 377 seperate of counteracting the IFN response.

378 NS1B also can directly bind to and block the interferon-stimulated 379 Ubiquitin-like ISG15 protein, which is naturally conjugated to newly 380 synthesized proteins [92]. By binding to ISG15, NS1B prevents activation of 381 ISG15 by the UBE1L enzyme, and this activity is mediated by the $\mathrm{N}$-terminus 382 of the NS1B protein $[87,93]$. Published evidence suggests that NS1B re383 localizes the bound ISG15 to the nuclear speckles [94]. NS1B specifically 384 interacts with human and non-human primate ISG15, but not with other 385 mammalian ISG15-like mouse or canine, implicating the ISG15-NS1B 386 interaction in the host-restriction of FluB [94]. 
388 NS1A, although the mechanism seems to be different for the two viruses. The 389 unique targeting of ISG15 suggests that this interferon stimulated gene would 390 otherwise play an important antiviral role against influenza B virus.

\section{The Nuclear Export Protein (or Non-Structural 2) Protein (NEP)}

The NEP protein of FluB interacts with the nuclear export machinery,

394 contains a Nuclear Export Signal (NES) sequence in its N-terminus and 395 possesses nuclear export activity [95]. Furthermore, it is produced in the late 396 stages of infection, initially accumulating in the nucleus, then being 397 transported to the cytoplasm and finally, in plasma membrane proximal 398 regions, the sites of virion budding. NEP can also be detected in virions as 399 part of the vRNP complex. Interestingly, in FluB NEP seems to interact 400 directly with the vRNP and BM1 as opposed to FluA where M1 acts as a 401 bridge between NEP and the vRNP [96]. Overall, FluB NEP appears to have a 402 similar function to its FluA counterpart, although its precise function is yet to 403 be determined.

\section{Non-coding Sequences}

406 The coding sequences of Flu viruses are flanked by stretches of non407 coding sequences in both termini. These sequences form the promoter that 408 drives viral replication and transcription and also contain packaging signals 409 [3]. In the specific context of FluB, they have shown to be important for vRNA 410 replication [97]. The sequences at the extreme of each terminus are highly 411 conserved. Specifically, the first 9 nucleotides of the 3' vRNA end (5'- 
412 AGCAGAAGC-3' in CDNA) and the first 10 nucleotides of the 5' vRNA end

413 (with the exception of position 6) (3'- TCATCxTTGT-5' in cDNA) are highly

414 conserved among segments and strains (Fig 1C). The sequences in the two

415 termini show a high degree of complementarity and thus form secondary RNA

416 structures. Between these extreme terminal sequences and the coding

417 sequences, lie non-coding sequences that are conserved for each segment

418 but differ between segments [98]. Understanding the non-coding sequences

419 of influenza viruses has been pivotal in the generation of reverse genetics 420 systems[2,98-100] as well as the design of novel vaccination strategies [101].

\section{Immunology of FluB}

\section{Innate Immune Recognition and Response}

As with other aspects of FluB immunology, the innate immune

425 response has been largely unexplored. The recognition receptors for FluB

426 have not been identified but RIG-I seems to be essential in the response to

427 FluB. MDA-5 might play an additional, although not essential, role in initiating

428 an innate response against FluB [102]. PKR can also detect viral RNAs and

429 inhibit replication of FluB both in cell culture and in mice, as highlighted by the

430 inhibition of PKR by NS1B. Activation of PKR was temporally linked to the

431 appearance of viral RNPs in the cytoplasm and purified vRNPs could activate

432 PKR activity in vitro [103]. FluB induces IFN-dependent and independent

433 responses through activation of IRF3 [104]. The MAP Kinase and NFKB

434 pathways can be activated. Interestingly, the innate immune recognition of

435 FluB occurs earlier in infection of dendritic cells than FluA (peak IFN- $\lambda$ mRNA

436 at 2-4 hours for FluB and at 8-16 hours for FluA) and is independent of viral 
replication or transcription [105]. The mechanism behind this early recognition

438 is not yet understood.

439 In response to in vitro FluB infection, epithelial and innate cells can

440 produce type I and III interferons, specifically IFN $\alpha$, IFN $\beta$, IFN $\lambda 1$, and other 441 cytokines/chemokines, including CXCL10, CXCL8, CCL5, CCL2, IL1- $\beta$, IL-18

442 and IL-6, although the specific levels and roles remain to be investigated [105443 107]. Similarly, although $\gamma \delta-T$ cell hybridomas can respond to both FluA- and 444 FluB-infected cells [108], the roles and functions of $\gamma \delta-T$ cells or other innate 445 immune cells in controlling FluB are unknown.

\section{Antibody Responses}

Similarly to FluA, the vast majority of antibody responses against FluB

449 are directed at the HA. The four main antigenic sites are in the globular head 450 of the HA1 and include the 120 loop (residues 116-137), the 150 loop 451 (residues 141-150), the 160 loop (residues 162-167) and the 190 loop 452 (residues 194-202) [42]. These sites of the HA are under positive selection, 453 indicating the effect of the antibody response to the evolution of the virus 454 [109]. Antibodies against the NA can also neutralize FluB and protect mice 455 from sub-lethal infections. Interestingly, immunization with B/Yamagata/16/88 456 NA can confer protection against B/Yamagata/16/87 as well as B/Victoria/2/87 457 and B/Malaysia/2506/04, both of which belong to the Victoria lineage [110]. 458 Although the two lineages are considered antigenically distinct, HA-directed 459 antibody cross-reactivity between the two lineages has been observed in 460 various contexts [111-115] potentially due to original antigenic $\sin [116]$. 
462 for FluB, firstly in a seminal paper by Dreyfus et al [117]. Antibody CR8033 463 binds to the RBS region of the HA, while antibody CR8071 binds to the 464 vestigial esterase domain at the base of the HA head. Both can neutralize 465 viruses from the two FluB lineages in vivo, even at low doses. Interestingly, 466 they can inhibit the release of viral progeny rather than the entry of viruses 467 into cells. Another antibody, CR9114, binds to the HA stem, on an epitope 468 conserved across FluB and FluA viruses. Structural studies showed that this 469 antibody binds to the $\mathrm{HA}$ of $\mathrm{H} 1, \mathrm{H} 3, \mathrm{H} 5, \mathrm{H} 7, \mathrm{H} 9$ and FluB. CR9114 blocks the $470 \mathrm{pH}$-induced conformational changes in $\mathrm{HA}$ and subsequent membrane fusion. 471 CR9114 could protect mice against lethal infection. Interestingly, sequence 472 analysis of the $V_{H}$ region of CR9114 showed a small number of somatic 473 mutations, suggesting that this broadly neutralizing antibody can be generated 474 from genomic sequences without extensive hypermutation [117]. Yasugi et al 475 [118] reported another broadly neutralizing antibody against FluB (5A7), which 476 targets the C-terminal region of HA1. This antibody showed therapeutic 477 efficacy even when administered after challenge. Broadly neutralizing 478 antibodies against the NA have also been reported. An antibody recognizing 479 residues 222-230 (N2 numbering), an epitope highly conserved across FluB 480 and FluA, can inhibit growth of FluB in cells but was not assessed in vivo $481[119,120]$. Vaccines that would prime antibody responses against such highly 482 conserved and cross-reactive epitopes would be of vital importance in 483 generating universal immunity against influenza viruses.

\section{T cells Responses}


been extensively studied in mice and human PBMCs [121-126]. However,

488 little is known about $\mathrm{T}$ cell responses to FluB. Mice lacking any antibody

489 responses are protected from lethal FluB challenge in a CD8 ${ }^{+}-\mathrm{T}^{-}$cell-

490 dependent manner. Primary infection can protect from secondary FluB

491 infection and this is also dependent on $\mathrm{CD}^{+}$as well as $\mathrm{CD}^{+} \mathrm{T}$ cells [127].

492 Thus, similarly to FluA, T cells have an important role in promoting recovery

493 from FluB infection. Three epitopes from NP of FluB have been identified for

$494 \mathrm{CD}^{+}$T cells. Robbins et al [128], identified the HLA-A*0201-restricted NP $82-94$

495 (MVVKLGEFYNQMM) epitope by its low similarity (leucine before a glycine

496 followed by hydrophobic residues) to the FluA A2+-M156-68 epitope. Two HLA-

497 B8 restricted epitopes from the NP protein of FluB (NP ${ }_{30-38}$ RPIIRPAT and

498 NP 263-271 ADRGLLRDI) were also identified later [129]. However, in a recent 499 study, only the $\mathrm{NP}_{30-38}$ peptide could induce INF $\gamma$ production from FluB 500 polyclonal CTLs [130]. It has also been proposed that CTL responses against

501 FluB are preferentially directed against HLA-B8 epitopes [131]. It was recently

502 shown that FluB-specific polyclonal CTLs cross-react with viruses from the 503 opposing lineage of FluB [130]. Some influenza A PB1 epitopes are 504 conserved even in FluB PB1 sequence [132], but whether T cells against 505 these epitopes are cross-reactive and cross-protective against both FluA and 506 FluB remains to be determined experimentally. Although primary infection with 507 FluB cannot protect from secondary infection with FluA and vice versa, in the 508 BALB/c mouse model $[133,134]$, immunization strategies that probe T-cell 509 responses against conserved and cross-reactive epitopes could potentially 510 confer universal heterologous immunity against Flu viruses. Thus, determining 
511 the cross-reactivity of $\mathrm{T}$ cells against FluA and FluB is a key step in

512 understanding universal anti-influenza T cell immunity.

\section{$514 \quad$ Flu B Vaccines}

515 Immunization against FluB is provided in the traditional Trivalent

516 Inactivated Vaccine (TIV). However, until recently the annual formulation of

517 this vaccine only contained a virus from either the Victoria or the Yamagata

518 lineage depending on the year (Table 1). The high levels of co-circulation of

519 the two lineages in the last decade results in frequent mismatch of predicted

520 and circulating strains and has necessitated the inclusion of viruses from both

521 lineages in the formulation of a quadrivalent vaccine [27]. The quadrivalent

522 vaccine is available as an inactivated intramuscular vaccine or as a LAIV in a 523 nasal spray.

$524 \quad$ High yields of virus are required during the production of inactivated 525 vaccines. For FluA, this is achieved by reassortment, resulting in the 526 combination of the antigenic surface proteins in the PR8 (A/Puerto Rico/8/34) 527 backbone, which grows to high titers during production in eggs. There is no 528 established 'high-yield' strain for FluB and the yield of FluB during annual 529 production varies greatly. Ways to generate high yields of FluB for vaccine 530 purposes as well as studies into the attenuation mechanisms of influenza B 531 LAIV have been reviewed [2]. Recently Le et al [135], generated reassortant 532 viruses with $\mathrm{B} / \mathrm{Lee} / 40$ as a donor strain and found that the NP gene from the $533 \mathrm{~B} /$ Lee/40 strain was central for high-yield production. However, overall there is 534 lack of a 'high-yield' FluB strain. 


\section{Antiviral Strategies against FluB}

Two classes of licensed anti-influenza drugs exist, adamantanes (eg.

538 amantadine and rimantadine) and NAls, like oseltamivir and zanamivir.

539 Adamantanes block the AM2 ion channel activity but not of BM2. This is

540 attributed to the polar amino aside residues in the BM2 ion channel cavity, as

541 they reduce accessibility of the drug to the ion channel pore [69].

542 Consequently, these drugs are ineffective against FluB.

543 NAls can inhibit FluB replication, although with variable efficiency. The

544 inhibitory effect of NAls against both FluA and FluB can be attributed to the

545 high conservation of the catalytic site across both FluA and FluB. The efficacy

546 of NAls and the resistance mutations of FluBs were extensively reviewed

547 recently by Burnham et al [46]. Overall, oseltamivir shows lower efficacy

548 against FluB than FluA, while zaramivir shows comparable efficacy.

549 Mutations conferring resistance to NAls have been reported in clinical

550 isolates, surveillance isolates as well as reverse genetics studies. These

551 mutations can be found either in the catalytic site of the enzyme (eg. R152K)

552 or in framework residues (eg D198N, I222T). Studies on the fitness cost of

553 NAl resistance mutations have shown that, contrary to most resistance

554 mutations, the E119A and $\mathrm{H} 274 \mathrm{Y}$ mutations have little impact on viral 555 replication and fitness [136,137]. Interestingly, it appears that different

556 mutations have different frequencies and effects in the NA gene from the two 557 FluB lineages [138]. Studies in NAI resistance by FluB highlight the possibility

558 of uncompromised NAI-resistant viruses emerging in human populations, 559 suggesting the need for increased surveillance and potentially novel antiviral 560 strategies. 


\section{Host Range of FluB}

564 outbreak in seals was reported in the Netherelands. Serological and

565 virological data indicated that a strain of FluB circulating in humans was 566 introduced to the seal population in 1995 [139] and further evidence of seal

567 FluB infections were reported later [140,141]. Sequence analysis of the

$568 \mathrm{~B} /$ Seal/1999 virus revealed high identity to a human FluB strain, suggesting

569 no adaptation of the virus in seals. A serological survey in swine farms across

570 the US revealed antibodies against FluB in pig serum samples but virus was

571 only detected by RT-PCR of the NS segment in a limited number of nasal

572 swabs. Unfortunately, virus could not be isolated. The partial sequences

573 derived from the RT-PCR were of high homology to human isolates,

574 suggesting no adaptation. In an experimental setting, FluB could replicate in

575 the respiratory tracts of pigs and infected animals exhibited flu-like symptoms,

576 lung lesions and seroconversion. FluB transmitted via direct contact from

577 infected to sentinel animals, although transmission was limited [142].

578 Antibodies against FluB have also been detected in guinea pigs [143], and

579 FluB replicates and transmits efficiently during experimental infection of 580 guinea pigs [144]. In addition, FluB replicates in ferret airway epithelial cells 581 as well as infected animals and transmits between ferrets, which are 582 considered the gold standard animal model for human influenza infections 583 [145](unpublished observations Elderfield, Koutsakos \& Barclay). Overall, 584 while endemic circulation of FluB is restricted to humans, there are indications 585 of FluB cross overs from humans to other species. However, there is currently 
no evidence of sustained animal reservoirs of FluB. As discussed above, this restriction of FluB in humans may, at least to an extent, be attributed to the human host specific interactions of NS1[5,87,94] and M1 [58] proteins.

589

\section{Conclusions \& Future Perspectives}

591

Over the last decade, it has become apparent that FluB constitutes a

592 substantial part of annual flu epidemics. The particular pathogenicity of FluB

593 in children, the co-circulation of the two lineages, which results in frequent

594 mismatches of vaccine and circulating strains, and the emergence of NAl-

595 resistance mutations with no apparent fitness cost further highlight the 596 importance of FluB as a human pathogen.

Although it has generally been assumed that FluA and FluB viruses are

598 similar, studies from the last decade emphasize multiple levels of uniqueness 599 in FluB virology, immunology and evolution (Table 2). Some FluB proteins, 600 like NP, have unique domains, others, like NB, are uniquely encoded by FluB 601 viruses and still others can work in unique ways, like the cap-snatching 602 mechanism of the FluB polymerases.

603 The numerous gaps of knowledge regarding FluB mean that multiple 604 avenues of research can prove fruitful in the future. A better understanding of 605 the evolution and phylodynamics of FluB viruses, such as the interactions 606 between the two lineages and the interactions between FluA and FluB, can 607 aid surveillance and vaccine strain determination and may inform 608 epidemiology [39]. A clearer picture of the molecular virology of FluB could 609 provide a better understanding of FluB pathogenicity, adaptation and design 610 of novel antiviral strategies. A more comprehensive understanding of FluB 
611 immunology would be an important step towards more effective vaccines and

612 possibly antibody- and/or $\mathrm{T}$ cell-mediated universal influenza immunity.

613 Overall, FluB is a research field waiting to explode.

\section{Executive Summary}

616

\section{Clinical Presentation and Epidemiology}

618

- FluB strains dominate every 2-4 years.

- Clinical presentations are similar to FluA, although usually more severe in children and adolescents.

\section{Evolution and Phylodynamics}

- Two lineages of FluB co-circulate globally, although usually one predominates in any given year..

- The two lineages exhibit contrasting phylodynamics.

- Re-assortment between the same lineage or two lineages contributes to the evolution of FluB.

\section{Virology}

630

631

632

633

634

635

636

637

638

639

- FluB has 8 gene segments encoding for 11 proteins.

- These generally have low sequence similarity to FluA and can exhibit important functional differences.

- NB is uniquely encoded by FluB and its function is unknown.

- NS1 inhibits the IFN $\alpha \beta$ response as well as ISG15 in a species-specific manner.

- The exact contributions of FluB proteins to pathogenicity and hostadaptation are unclear.

\section{Immunology}

- Mainly unexplored.

- FluB stimulates IFN $\alpha \beta$ and cytokine antiviral responses, although with kinetics different to FluA.

- Antibody responses are directed against HA and universally neutralizing antibodies against $\mathrm{HA}$ have been identified.

- T cells confer protection against primary and secondary infection in a mouse model.

- Only a handful $\mathrm{CD}^{+} \mathrm{T}$ cell epitopes have been identified and T cell cross-reactivity between two lineages has been described.

- Trivalent (two FluA and one FluB strain) and quadrivalent (two FluA and two FluB strains) vaccines are available and updated annually. 


\section{Antiviral Strategies}

654

655

656

657

658

659

- Adamantanes are ineffective against FluB.

- Neuranimidase inhibitors work but not as efficiently as for FluA.

- Resistance mutations with no apparent fitness cost have been detected.

\section{Host Range}

660

661

662

663

664

665

References

666

667

1. Francis T, Jr. A New Type of Virus from Epidemic Influenza. Science,

668

669

2. Jackson D, Elderfield RA, Barclay WS. Molecular studies of influenza B virus in the reverse genetics era. The Journal of General Virology, 92(Pt 1), 1-17 (2011).

** Thorough review of the use of reverse genetics to study Influenza B viruses.

675

3. Peter Palese MLS. Orthomyxoviridae:The Viruese and their

4. Rota PA, Wallis TR, Harmon MW, Rota JS, Kendal AP, Nerome K. Cocirculation of two distinct evolutionary lineages of influenza type $B$ virus since 1983. Virology, 175(1), 59-68 (1990).

5. McCullers JA, Saito T, Iverson AR. Multiple genotypes of influenza B virus circulated between 1979 and 2003. Journal of virology, 78(23), 12817-12828 (2004).

6. Vijaykrishna D, Holmes EC, Joseph $U$ et al. The contrasting phylodynamics of human influenza B viruses. eLife, 4, e05055 (2015). 
${ }^{* *}$ Detailed analysis of the phylogenetics and evolution of the two lineages.

7. Thompson WW, Shay DK, Weintraub E et al. Mortality associated with influenza and respiratory syncytial virus in the United States. Jama, 289(2), 179-186 (2003).

8. Lin YP, Gregory V, Bennett M, Hay A. Recent changes among human influenza viruses. Virus research, 103(1-2), 47-52 (2004).

9. W. Paul Glezen JKS, Carrie M. Kuehn, Kellie J. Ryan, and John Oxford. The Burden of Influenza B: A Structured Literature Review. American Journal of Public Health, 103(3), e43-e51 (2013).

* Interesting meta-analysis of Influenza B clinical data.

10. Gutierrez-Pizarraya A, Perez-Romero P, Alvarez R et al. Unexpected severity of cases of influenza B infection in patients that required hospitalization during the first postpandemic wave. The Journal of infection, 65(5), 423-430 (2012).

11. Su S, Chaves SS, Perez A et al. Comparing clinical characteristics between hospitalized adults with laboratory-confirmed influenza a and $B$ virus infection. Clinical infectious diseases : an official publication of the Infectious Diseases Society of America, 59(2), 252-255 (2014).

12. Thompson WW, Moore MR, Weintraub E et al. Estimating influenzaassociated deaths in the United States. Am J Public Health, 99 Suppl 2, S225-230 (2009).

13. Matias G, Taylor R, Haguinet F, Schuck-Paim C, Lustig R, Shinde V. Estimates of mortality attributable to influenza and RSV in the United States during 1997-2009 by influenza type or subtype, age, cause of death, and risk status. Influenza and other respiratory viruses, 8(5), 507-515 (2014).

14. Kaczmarek M, Owen R, Barr IG. Annual report of the National Influenza Surveillance Scheme, 2008. Communicable diseases intelligence quarterly report, 34(1), 8-22 (2010).

15. Pubic Health England. Surveillance of influenza and other respiratory viruses, including novel respiratory viruses, in the United Kingdom: Winter 2012/13. (2013) 
16. Tewawong N, Suwannakarn K, Prachayangprecha S et al. Molecular epidemiology and phylogenetic analyses of influenza $B$ virus in Thailand during 2010 to 2014. PloS one, 10(1), e0116302 (2015).

17. Peter F. Wright GN, Yoshihiro Kawaoka. Orthomixoviruses. In: Fields Virology. David M. Knipe, PMH (Ed. (LIPPINCOT WILLIAMS \& WILKINS, a WOLTERS KLUWER BUSINESS, USA, 2007)

18. Irving SA, Patel DC, Kieke BA et al. Comparison of clinical features and outcomes of medically attended influenza $A$ and influenza $B$ in a defined population over four seasons: 2004-2005 through 2007-2008. Influenza and other respiratory viruses, 6(1), 37-43 (2012).

19. Moon JH, $\mathrm{Na} \mathrm{JY}, \mathrm{Kim} \mathrm{JH}$ et al. Neurological and muscular manifestations associated with influenza B infection in children. Pediatric neurology, 49(2), 97-101 (2013).

20. Thabet FI, Khalil S, Naz F, Dyme IZ. Cerebellar mutism and reversible cytotoxic edema in influenza B-associated encephalopathy. Pediatric neurology, 49(6), 489-492 (2013).

21. Frank H, Wittekind C, Liebert UG et al. Lethal influenza B myocarditis in a child and review of the literature for pediatric age groups. Infection, 38(3), 231-235 (2010).

22. Paddock CD, Liu L, Denison AM et al. Myocardial injury and bacterial pneumonia contribute to the pathogenesis of fatal influenza B virus infection. The Journal of infectious diseases, 205(6), 895-905 (2012).

23. Taremi M, Amoroso A, Nace HL, Gilliam BL. Influenza B-induced refractory cardiogenic shock: a case report. BMC infectious diseases, 13, 452 (2013).

24. Aebi T, Weisser M, Bucher E, Hirsch HH, Marsch S, Siegemund M. Co-infection of Influenza B and Streptococci causing severe pneumonia and septic shock in healthy women. BMC infectious diseases, 10, 308 (2010).

25. Scaber J, Saeed S, Ihekweazu C, Efstratiou A, McCarthy N, O'Moore E. Group A streptococcal infections during the seasonal influenza outbreak 2010/11 in South East England. Euro surveillance : bulletin Europeen sur les maladies transmissibles = European communicable disease bulletin, 16(5) (2011). 
26. Erhard van der Vries DKMI, Benjamin J. Cowling, Jitao D. Zhang, Xiao Tong, Krzysztof Wojtowicz, Martin Schutten, and Charles A. Boucher. Outcomes and Susceptibility to Neuraminidase Inhibitors in Individuals Infected with Different Influenza B Lineages: the Influenza Resistance Information Study. Journal of Infectious Diseases, (2015).

27. Belshe RB. The need for quadrivalent vaccine against seasonal influenza. Vaccine, 28 Suppl 4, D45-53 (2010).

28. Hite LK, Glezen WP, Demmler GJ, Munoz FM. Medically attended pediatric influenza during the resurgence of the Victoria lineage of influenza B virus. International journal of infectious diseases : IJID : official publication of the International Society for Infectious Diseases, 11(1), 40-47 (2007).

29. Olson DR, Heffernan RT, Paladini M, Konty K, Weiss D, Mostashari F. Monitoring the impact of influenza by age: emergency department fever and respiratory complaint surveillance in New York City. PLoS medicine, 4(8), e247 (2007).

30. Socan M, Prosenc K, Ucakar V, Berginc N. A comparison of the demographic and clinical characteristics of laboratory-confirmed influenza B Yamagata and Victoria lineage infection. Journal of clinical virology : the official publication of the Pan American Society for Clinical Virology, 61(1), 156-160 (2014).

31. Tan Y, Guan W, Lam TT et al. Differing epidemiological dynamics of influenza B virus lineages in Guangzhou, southern China, 2009-2010. Journal of virology, 87(22), 12447-12456 (2013).

32. Wang YF, Chang CF, Chi CY, Wang HC, Wang JR, Su IJ. Characterization of glycan binding specificities of influenza B viruses with correlation with hemagglutinin genotypes and clinical features. Journal of medical virology, 84(4), 679-685 (2012).

33. Skowronski DM, Janjua NZ, Sabaiduc S et al. Influenza A/subtype and B/lineage effectiveness estimates for the 2011-2012 trivalent vaccine: cross-season and cross-lineage protection with unchanged vaccine. The Journal of infectious diseases, 210(1), 126-137 (2014).

34. Chen R, Holmes EC. The evolutionary dynamics of human influenza B virus. Journal of molecular evolution, 66(6), 655-663 (2008). 
** Thorough analysis of Influenza B evolution and epidemiology over and extended time period.

35. Bedford T, Riley S, Barr IG et al. Global circulation patterns of seasonal influenza viruses vary with antigenic drift. Nature, 523(7559), 217-220 (2015).

** Detailed comparative analysis of Influenza A and B global spatio-temporal patterns over 12 years.

36. Patterson Ross Z, Komadina N, Deng YM et al. Inter-Seasonal Influenza is Characterized by Extended Virus Transmission and Persistence. PLoS pathogens, 11(6), e1004991 (2015).

37. Nelson MI, Simonsen L, Viboud C et al. Stochastic processes are key determinants of short-term evolution in influenza a virus. PLoS pathogens, 2(12), e125 (2006).

38. Dudas G, Bedford T, Lycett S, Rambaut A. Reassortment between influenza $B$ lineages and the emergence of a coadapted PB1-PB2-HA gene complex. Molecular biology and evolution, 32(1), 162-172 (2015).

** Interesting bionformatics analysis of Influenza B reassortment patterns over 28 years.

39. Laurie KL, Guarnaccia TA, Carolan LA et al. Interval Between Infections and Viral Hierarchy Are Determinants of Viral Interference Following Influenza Virus Infection in a Ferret Model. The Journal of infectious diseases, (2015).

40. Wang $Q$, Tian X, Chen X, Ma J. Structural basis for receptor specificity of influenza B virus hemagglutinin. Proceedings of the National Academy of Sciences of the United States of America, 104(43), 1687416879 (2007).

41. Matrosovich MN, Gambaryan AS, Tuzikov $A B$ et al. Probing of the receptor-binding sites of the $\mathrm{H} 1$ and $\mathrm{H} 3$ influenza $A$ and influenza $B$ virus hemagglutinins by synthetic and natural sialosides. Virology, 196(1), 111-121 (1993).

42. Wang Q, Cheng F, Lu M, Tian X, Ma J. Crystal structure of unliganded influenza B virus hemagglutinin. Journal of virology, 82(6), 3011-3020 (2008). 
43. Ni F, Mbawuike IN, Kondrashkina E, Wang Q. The roles of hemagglutinin Phe-95 in receptor binding and pathogenicity of influenza B virus. Virology, 450-451, 71-83 (2014).

44. Medina RA, Garcia-Sastre A. Influenza A viruses: new research developments. Nature reviews. Microbiology, 9(8), 590-603 (2011).

45. Burmeister WP, Ruigrok RW, Cusack S. The 2.2 A resolution crystal structure of influenza B neuraminidase and its complex with sialic acid. The EMBO journal, 11(1), 49-56 (1992).

50. Demers A, Ran Z, Deng $Q$ et al. Palmitoylation is required for

52. Shimbo K, Brassard DL, Lamb RA, Pinto LH. Viral and cellular small Xenopus oocytes. Biophysical journal, 69(5), 1819-1829 (1995). 
851 53. Ewart GD, Sutherland T, Gage PW, Cox GB. The Vpu protein of human immunodeficiency virus type 1 forms cation-selective ion channels. Journal of virology, 70(10), 7108-7115 (1996).

54. Sunstrom NA, Premkumar LS, Premkumar A, Ewart G, Cox GB, Gage 855 PW. Ion channels formed by NB, an influenza B virus protein. The Journal of membrane biology, 150(2), 127-132 (1996).

55. Fischer WB, Pitkeathly M, Wallace BA, Forrest LR, Smith GR, Sansom MS. Transmembrane peptide NB of influenza B: a simulation, structure, and conductance study. Biochemistry, 39(41), 12708-12716 (2000).

56. Lamb RA, Pinto LH. Do Vpu and Vpr of human immunodeficiency virus

57. Betakova T, Hay AJ. Evidence that the CM2 protein of influenza C

58. McCullers JA, Hoffmann E, Huber VC, Nickerson AD. A single amino

60. Briedis DJ, Lamb RA, Choppin PW. Sequence of RNA segment 7 of

61. Paterson RG, Takeda M, Ohigashi Y, Pinto LH, Lamb RA. Influenza B

62. Odagiri T, Hong J, Ohara Y. The BM2 protein of influenza B virus is

59. Cao S, Jiang J, Li J et al. Characterization of the nucleocytoplasmic shuttle of the matrix protein of influenza B virus. Journal of virology, 88(13), 7464-7473 (2014). the influenza $B$ virus genome: partial amino acid homology between the membrane proteins (M1) of influenza $A$ and $B$ viruses and conservation of a second open reading frame. Virology, 116(2), 581588 (1982). virus BM2 protein is an oligomeric integral membrane protein expressed at the cell surface. Virology, 306(1), 7-17 (2003). synthesized in the late phase of infection and incorporated into virions as a subviral component. The Journal of general virology, 80 ( $\mathrm{Pt} 10)$, 2573-2581 (1999). 
885

886

887

888

889

890

891

892

893

894

895

896

897

898

899

900

901

902

903

904

905

906

907

908

909

910

911

912

913

914

915

916

917

918

63. Watanabe S, Imai M, Ohara $\mathrm{Y}$, Odagiri T. Influenza B Virus BM2 Protein Is Transported through the trans-Golgi Network as an Integral Membrane Protein. Journal of virology, 77(19), 10630-10637 (2003).

64. Wang J, Pielak RM, McClintock MA, Chou JJ. Solution structure and functional analysis of the influenza B proton channel. Nature structural \& molecular biology, 16(12), 1267-1271 (2009).

* Solution of NMR strucutre of BM2 protein.

65. Imai M, Watanabe S, Ninomiya A, Obuchi M, Odagiri T. Influenza B virus BM2 protein is a crucial component for incorporation of viral ribonucleoprotein complex into virions during virus assembly. Journal of virology, 78(20), 11007-11015 (2004).

66. Jackson D, Zurcher T, Barclay W. Reduced incorporation of the influenza B virus BM2 protein in virus particles decreases infectivity. Virology, 322(2), 276-285 (2004).

67. Hatta M, Goto H, Kawaoka Y. Influenza B virus requires BM2 protein for replication. Journal of virology, 78(11), 5576-5583 (2004).

68. Mould JA, Paterson RG, Takeda M et al. Influenza B Virus BM2 Protein Has Ion Channel Activity that Conducts Protons across Membranes. Developmental Cell, 5(1), 175-184 (2003).

69. Ma C, Soto CS, Ohigashi $\mathrm{Y}$ et al. Identification of the pore-lining residues of the BM2 ion channel protein of influenza $B$ virus. The Journal of biological chemistry, 283(23), 15921-15931 (2008).

70. Zhang $\mathrm{H}, \mathrm{Yu} \mathrm{H}$, Wang $\mathrm{J}$ et al. The BM2 protein of influenza B virus interacts with p53 and inhibits its transcriptional and apoptotic activities. Molecular and cellular biochemistry, 403(1-2), 187-197 (2015).

71. Horvath CM, Williams MA, Lamb RA. Eukaryotic coupled translation of tandem cistrons: identification of the influenza B virus BM2 polypeptide. The EMBO journal, 9(8), 2639-2647 (1990).

72. Hatta M, Kohlmeier CK, Hatta Y, Ozawa M, Kawaoka Y. Region required for protein expression from the stop-start pentanucleotide in the M gene of influenza B virus. Journal of virology, 83(11), 5939-5942 (2009).

73. Powell ML, Napthine S, Jackson RJ, Brierley I, Brown TD. Characterization of the termination-reinitiation strategy employed in the 

(2008).

74. Powell ML, Leigh KE, Poyry TA, Jackson RJ, Brown TD, Brierley I. Further characterisation of the translational termination-reinitiation (2011).

75. Stevens MP, Barclay WS. The N-terminal extension of the influenza B virus nucleoprotein is not required for nuclear accumulation or the expression and replication of a model RNA. Journal of virology, 72(6), 5307-5312 (1998).

76. $\mathrm{Ng} \mathrm{AK}$, Lam MK, Zhang $\mathrm{H}$ et al. Structural basis for RNA binding and homo-oligomer formation by influenza B virus nucleoprotein. Journal of virology, 86(12), 6758-6767 (2012).

77. Shen YF, Chen YH, Chu SY et al. E339...R416 salt bridge of nucleoprotein as a feasible target for influenza virus inhibitors. Proceedings of the National Academy of Sciences of the United States of America, 108(40), 16515-16520 (2011).

78. Wanitchang A, Narkpuk J, Jongkaewwattana A. Nuclear import of influenza B virus nucleoprotein: involvement of an $\mathrm{N}$-terminal nuclear localization signal and a cleavage-protection motif. Virology, 443(1), 59-68 (2013).

79. Sherry L, Smith M, Davidson S, Jackson D. The $N$ terminus of the influenza B virus nucleoprotein is essential for virus viability, nuclear localization, and optimal transcription and replication of the viral genome. Journal of virology, 88(21), 12326-12338 (2014).

80. Reich S, Guilligay D, Pflug A et al. Structural insight into cap-snatching and RNA synthesis by influenza polymerase. Nature, 516(7531), 361366 (2014).

\section{${ }^{* *}$ First crystal structure of Influenza heterotrimeric polymerase complex.}

81. Deng $Q$, Wang $D$, Xiang $X$ et al. Nuclear localization of influenza $B$ polymerase proteins and their binary complexes. Virus research, 156(1-2), 49-53 (2011).

82. Wakai C, Iwama M, Mizumoto K, Nagata K. Recognition of cap structure by influenza B virus RNA polymerase is less dependent on 

the methyl residue than recognition by influenza $A$ virus polymerase. Journal of virology, 85(15), 7504-7512 (2011).

83. Liu Y, Yang Y, Fan J, He R, Luo M, Zheng X. The crystal structure of the PB2 cap-binding domain of influenza B virus reveals a novel cap recognition mechanism. The Journal of biological chemistry, 290(14), 9141-9149 (2015).

84. Cauldwell AV, Long JS, Moncorge O, Barclay WS. Viral determinants of influenza A virus host range. The Journal of general virology, 95( $\mathrm{Pt}$ 6), 1193-1210 (2014).

85. Dauber B, Heins G, Wolff T. The Influenza B Virus Nonstructural NS1 Protein Is Essential for Efficient Viral Growth and Antagonizes Beta Interferon Induction. Journal of virology, 78(4), 1865-1872 (2004).

86. Hai R, Martinez-Sobrido L, Fraser KA, Ayllon J, Garcia-Sastre A, Palese P. Influenza B virus NS1-truncated mutants: live-attenuated vaccine approach. Journal of virology, 82(21), 10580-10590 (2008).

87. Guan R, Ma LC, Leonard PG et al. Structural basis for the sequencespecific recognition of human ISG15 by the NS1 protein of influenza B virus. Proceedings of the National Academy of Sciences of the United States of America, 108(33), 13468-13473 (2011).

88. Schneider J, Dauber B, Melen K, Julkunen I, Wolff T. Analysis of influenza B Virus NS1 protein trafficking reveals a novel interaction with nuclear speckle domains. Journal of virology, 83(2), 701-711 (2009).

89. Noah DL, Twu KY, Krug RM. Cellular antiviral responses against influenza $A$ virus are countered at the posttranscriptional level by the viral NS1A protein via its binding to a cellular protein required for the $3^{\prime}$ end processing of cellular pre-mRNAS. Virology, 307(2), 386-395 (2003).

90. Donelan NR, Dauber B, Wang X, Basler CF, Wolff T, Garcia-Sastre A. The $\mathrm{N}$ - and C-terminal domains of the NS1 protein of influenza B virus can independently inhibit IRF-3 and beta interferon promoter activation. Journal of virology, 78(21), 11574-11582 (2004).

91. Dauber B, Schneider J, Wolff T. Double-stranded RNA binding of influenza B virus nonstructural NS1 protein inhibits protein kinase R but 
is not essential to antagonize production of alpha/beta interferon. Journal of virology, 80(23), 11667-11677 (2006).

92. Iwasaki A, Pillai PS. Innate immunity to influenza virus infection. Nature reviews. Immunology, 14(5), 315-328 (2014).

93. Yuan W, Krug RM. Influenza B virus NS1 protein inhibits conjugation of the interferon (IFN)-induced ubiquitin-like ISG15 protein. The EMBO

94. Sridharan H, Zhao C, Krug RM. Species specificity of the NS1 protein 994

96. Imai M, Watanabe S, Odagiri T. Influenza B virus NS2, a nuclear of influenza B virus: NS1 binds only human and non-human primate ubiquitin-like ISG15 proteins. The Journal of biological chemistry, 285(11), 7852-7856 (2010).

95. Paragas J, Talon J, O'Neill RE, Anderson DK, Garcia-Sastre A, Palese P. Influenza B and C virus NEP (NS2) proteins possess nuclear export activities. Journal of virology, 75(16), 7375-7383 (2001).

export protein, directly associates with the viral ribonucleoprotein complex. Archives of virology, 148(10), 1873-1884 (2003).

97. Barclay WS, Palese P. Influenza B viruses with site-specific mutations introduced into the HA gene. Journal of virology, 69(2), 1275-1279 (1995).

98. Zhou B, Lin X, Wang W et al. Universal influenza B virus genomic amplification facilitates sequencing, diagnostics, and reverse genetics. Journal of clinical microbiology, 52(5), 1330-1337 (2014).

99. Hoffmann E, Mahmood K, Yang CF, Webster RG, Greenberg HB, Kemble G. Rescue of influenza B virus from eight plasmids. Proceedings of the National Academy of Sciences of the United States of America, 99(17), 11411-11416 (2002).

100. Jackson D, Cadman A, Zurcher T, Barclay WS. A Reverse Genetics Approach for Recovery of Recombinant Influenza B Viruses Entirely from cDNA. Journal of virology, 76(22), 11744-11747 (2002).

101. Baker SF, Nogales A, Finch $C$ et al. Influenza A and B virus intertypic reassortment through compatible viral packaging signals. Journal of virology, 88(18), 10778-10791 (2014). 
102. Loo YM, Fornek J, Crochet $\mathrm{N}$ et al. Distinct RIG-I and MDA5 signaling by RNA viruses in innate immunity. Journal of virology, 82(1), 335-345 (2008).

103. Dauber B, Martinez-Sobrido L, Schneider $\mathrm{J}$ et al. Influenza B virus ribonucleoprotein is a potent activator of the antiviral kinase PKR. PLoS pathogens, 5(6), e1000473 (2009).

104. Kim MJ, Latham AG, Krug RM. Human influenza viruses activate an interferon-independent transcription of cellular antiviral genes: outcome with influenza A virus is unique. Proceedings of the National Academy of Sciences of the United States of America, 99(15), 10096-10101 (2002).

105. Osterlund $P$, Strengell $M$, Sarin LP et al. Incoming influenza $A$ virus evades early host recognition, while influenza B virus induces interferon expression directly upon entry. Journal of virology, 86(20), 11183-11193 (2012).

*This study pinpoints intersting differences in innate recognition between FluA and FluB.

106. Veckman V, Osterlund $\mathrm{P}$, Fagerlund $\mathrm{R}$, Melen $\mathrm{K}$, Matikainen S, Julkunen I. TNF-alpha and IFN-alpha enhance influenza-A-virusinduced chemokine gene expression in human A549 lung epithelial cells. Virology, 345(1), 96-104 (2006).

107. Ichinohe T, Pang IK, Iwasaki A. Influenza virus activates inflammasomes via its intracellular M2 ion channel. Nature immunology, 11(5), 404-410 (2010).

108. Ponniah S, Doherty PC, Eichelberger M. Selective response of gamma delta T-cell hybridomas to orthomyxovirus-infected cells. Journal of virology, 70(1), 17-22 (1996).

109. Nunes B, Pechirra P, Coelho A, Ribeiro C, Arraiolos A, Rebelo-deAndrade $\mathrm{H}$. Heterogeneous selective pressure acting on influenza $B$ Victoria- and Yamagata-like hemagglutinins. Journal of molecular evolution, 67(4), 427-435 (2008).

110. Wohlbold TJ, Nachbagauer $\mathrm{R}, \mathrm{Xu} \mathrm{H}$ et al. Vaccination with adjuvanted recombinant neuraminidase induces broad heterologous, but not 
heterosubtypic, cross-protection against influenza virus infection in mice. mBio, 6(2), e02556 (2015).

111. Levandowski RA, Gross PA, Weksler M, Staton E, Williams MS, Bonelli

112. Janjua NZ, Skowronski DM, De Serres G et al. Estimates of influenza vaccine effectiveness for 2007-2008 from Canada's sentinel surveillance system: cross-protection against major and minor variants. The Journal of infectious diseases, 205(12), 1858-1868 (2012).

1062

114. Walter EB, Neuzil KM, Zhu Y et al. Influenza vaccine immunogenicity in 1065

115. Ohmit SE, Thompson MG, Petrie JG et al. Influenza vaccine

116. Skowronski DM, Hottes TS, De Serres G et al. Influenza B/Victoria

113. Englund JA, Walter EB, Gbadebo A, Monto AS, Zhu Y, Neuzil KM. Immunization with trivalent inactivated influenza vaccine in partially immunized toddlers. Pediatrics, 118(3), e579-585 (2006).

6- to 23-month-old children: are identical antigens necessary for priming? Pediatrics, 118(3), e570-578 (2006). effectiveness in the 2011-2012 season: protection against each circulating virus and the effect of prior vaccination on estimates. Clinical infectious diseases : an official publication of the Infectious Diseases Society of America, 58(3), 319-327 (2014). antigen induces strong recall of B/Yamagata but lower B/Victoria response in children primed with two doses of B/Yamagata. The Pediatric infectious disease journal, 30(10), 833-839 (2011).

117. Dreyfus C, Laursen NS, Kwaks T et al. Highly conserved protective epitopes on influenza B viruses. Science, 337(6100), 1343-1348 (2012).

${ }^{* *}$ First report of highly conserved and cross-reactive antibody epitopes of Influenza B, includes crystal strucutres.

118. Yasugi M, Kubota-Koketsu R, Yamashita A et al. Human monoclonal antibodies broadly neutralizing against influenza $B$ virus. PLoS pathogens, 9(2), e1003150 (2013).

119. Gravel C, Li C, Wang J et al. Qualitative and quantitative analyses of virtually all subtypes of influenza $A$ and $B$ viral neuraminidases using 
antibodies targeting the universally conserved sequences. Vaccine, 28(36), 5774-5784 (2010).

120. Doyle TM, Li C, Bucher DJ et al. A monoclonal antibody targeting a highly conserved epitope in influenza B neuraminidase provides protection against drug resistant strains. Biochemical and biophysical research communications, 441(1), 226-229 (2013).

121. McMichael AJ, Gotch FM, Noble GR, Beare PA. Cytotoxic T-cell immunity to influenza. The New England journal of medicine, 309(1), 13-17 (1983).

122. Gras S, Kedzierski L, Valkenburg SA et al. Cross-reactive CD8+ T-cell immunity between the pandemic H1N1-2009 and H1N1-1918 influenza A viruses. Proceedings of the National Academy of Sciences of the United States of America, 107(28), 12599-12604 (2010).

123. Sridhar S, Begom S, Bermingham A et al. Cellular immune correlates of protection against symptomatic pandemic influenza. Nature medicine, 19(10), 1305-1312 (2013).

124. Quinones-Parra S, Grant E, Loh L et al. Preexisting CD8+ T-cell immunity to the H7N9 influenza A virus varies across ethnicities. Proceedings of the National Academy of Sciences of the United States of America, 111(3), 1049-1054 (2014).

125. Wang Z, Wan Y, Qiu C et al. Recovery from severe H7N9 disease is associated with diverse response mechanisms dominated by CD8(+) T cells. Nature communications, 6, 6833 (2015).

126. Hillaire ML, van Trierum SE, Kreijtz JH et al. Cross-protective immunity against influenza pH1N1 2009 viruses induced by seasonal influenza A (H3N2) virus is mediated by virus-specific T-cells. The Journal of general virology, 92(Pt 10), 2339-2349 (2011).

127. Epstein SL, Lo CY, Misplon JA, Bennink JR. Mechanism of protective immunity against influenza virus infection in mice without antibodies. Journal of immunology, 160(1), 322-327 (1998).

${ }^{*}$ First and, to our knowledge, only study on in vivo role of $\mathrm{T}$ cell during FluB infection.

128. P A Robbins LAL, P Rota, J Santos-Aguado, J Rothbard, A J McMichael, and J L Strominger. Comparison between two peptide 
epitopes presented to cytotoxic T lymphocytes by HLA-A2. Evidence for discrete locations within HLA-A2. J Immunology, 143(4089), 103 (1989).

1123

1124

1125

1126

1127

1128

1129

1130

1131

1132

1133

1134

1135

1136

1137

1138

1139

1140

1141

1142

1143

1144

1145

1146

1147

1148

1149

1150

1151

1152

1153

129. Robbins PA, Rota PA, Shapiro SZ. A broad cytotoxic T lymphocyte response to influenza type $B$ virus presented by multiple HLA molecules. International immunology, 9(6), 815-823 (1997).

130. van de Sandt CE, Dou Y, Vogelzang-van Trierum SE et al. Influenza B virus-specific CD8+ $T$ lymphocytes strongly cross-react with viruses of the opposing influenza B lineage. The Journal of general virology, (2015).

131. Boon AC, De Mutsert G, Fouchier RA, Sintnicolaas K, Osterhaus AD, Rimmelzwaan GF. Preferential HLA usage in the influenza virusspecific CTL response. Journal of immunology, 172(7), 4435-4443 (2004).

132. Masanori Terajima JABB, Mary Dawn $T$ Co and Francis A Ennis. Cross-reactive human $B$ cell and $T$ cell epitopes between influenza $A$ and B viruses. Virology Journal 10(244) (2013).

133. Epstein SL, Lo CY, Misplon JA et al. Mechanisms of heterosubtypic immunity to lethal influenza A virus infection in fully immunocompetent, $\mathrm{T}$ cell-depleted, beta2-microglobulin-deficient, and $\mathrm{J}$ chain-deficient mice. Journal of immunology, 158(3), 1222-1230 (1997).

134. Benton KA, Misplon JA, Lo CY, Brutkiewicz RR, Prasad SA, Epstein SL. Heterosubtypic Immunity to Influenza A Virus in Mice Lacking IgA, All Ig, NKT Cells, or T Cells. The Journal of Immunology, 166(12), 7437-7445 (2001).

135. Le J, Orff EJ, Fulvini AA et al. Development of high yield reassortants for influenza type B viruses and analysis of their gene compositions. Vaccine, 33(7), 879-884 (2015).

136. Burnham AJ, Armstrong J, Lowen AC, Webster RG, Govorkova EA. Competitive fitness of influenza $B$ viruses with neuraminidase inhibitorresistant substitutions in a coinfection model of the human airway epithelium. Journal of virology, 89(8), 4575-4587 (2015).

137. Burnham AJ, Baranovich $T$, Marathe BM, Armstrong J, Webster RG, Govorkova EA. Fitness costs for Influenza B viruses carrying 
neuraminidase inhibitor-resistant substitutions: underscoring the importance of $\mathrm{E} 119 \mathrm{~A}$ and $\mathrm{H} 274 \mathrm{Y}$. Antimicrobial agents and chemotherapy, 58(5), 2718-2730 (2014).

138. Farrukee $\mathrm{R}$, Leang $\mathrm{SK}$, Butler $\mathrm{J}$ et al. Influenza viruses with $\mathrm{B} /$ Yamagata- and B/Victoria-like neuraminidases are differentially affected by mutations that alter antiviral susceptibility. The Journal of antimicrobial chemotherapy, 70(7), 2004-2012 (2015).

139. A. D. M. E. Osterhaus GFR, B. E. E. Martina, T. M. Bestebroer, and R. A. M. Fouchier. Influenza B Virus in Seals. Science, 288(5468), 10511053 (2000).

${ }^{* *}$ First record of Influenza $B$ in a non-human host.

140. Andrea Blanc DR, Mario Clara, Federico Achaval, Alfredo Le Bas, and Juan Arbiza Serologic Evidence of Influenza A and B Viruses in South American Fur Seals (Arctocephalus australis). Journal of Wildlife Diseases, 45(2), 519-521 (2009).

141. Bodewes $R$, van de Bildt MW, van Elk CE et al. No serological evidence that harbour porpoises are additional hosts of influenza $B$ viruses. PloS one, 9(2), e89058 (2014).

142. Ran $Z$, Shen $\mathrm{H}$, Lang $\mathrm{Y}$ et al. Domestic pigs are susceptible to infection with influenza B viruses. Journal of virology, 89(9), 4818-4826 (2015).

143. Leyva-Grado VH, Mubareka S, Krammer F, Cardenas WB, Palese P. Influenza virus infection in guinea pigs raised as livestock, Ecuador. Emerging infectious diseases, 18(7), 1135-1138 (2012).

144. Pica N, Chou YY, Bouvier NM, Palese P. Transmission of influenza B viruses in the guinea pig. Journal of virology, 86(8), 4279-4287 (2012).

*Establishment of a FluB transmission system in guinea pigs.

145. Elderfield RA, Parker L, Stilwell P, Roberts KL, Schepelmann S, Barclay WS. Ferret airway epithelial cell cultures support efficient replication of influenza B virus but not Mumps virus. The Journal of general virology, (2015).

Acknowledgements 
1187 MK is supported by a University of Melbourne International Fee Remission 1188 Scholarship (MIFRS) and a University of Melbourne International Research 1189 Scholarships (MIRS). KK is an NHMRC CDF Level2 Fellow. The authors 1190 declare no conflict of interest.

1193 Figure Legends

1195 Figure 1. Overview of Influenza B viruses. A) Schematic of FluB virus. B)

1196 Genome organization of FluB. C) Non-coding sequences of FluB.

1198 Table 1. Vaccines strains of FluB in the Northern and Southern Hemispheres. 1199 QV=Quadrivalent Vaccine, N/A= Not applicable.

1201 Table 2. Comparison of FluA and FluB. 
A)

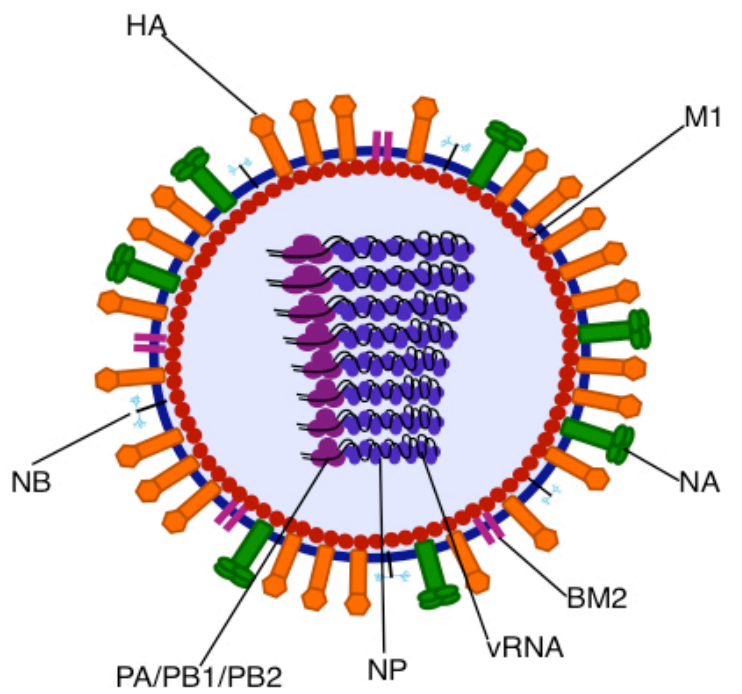

B)

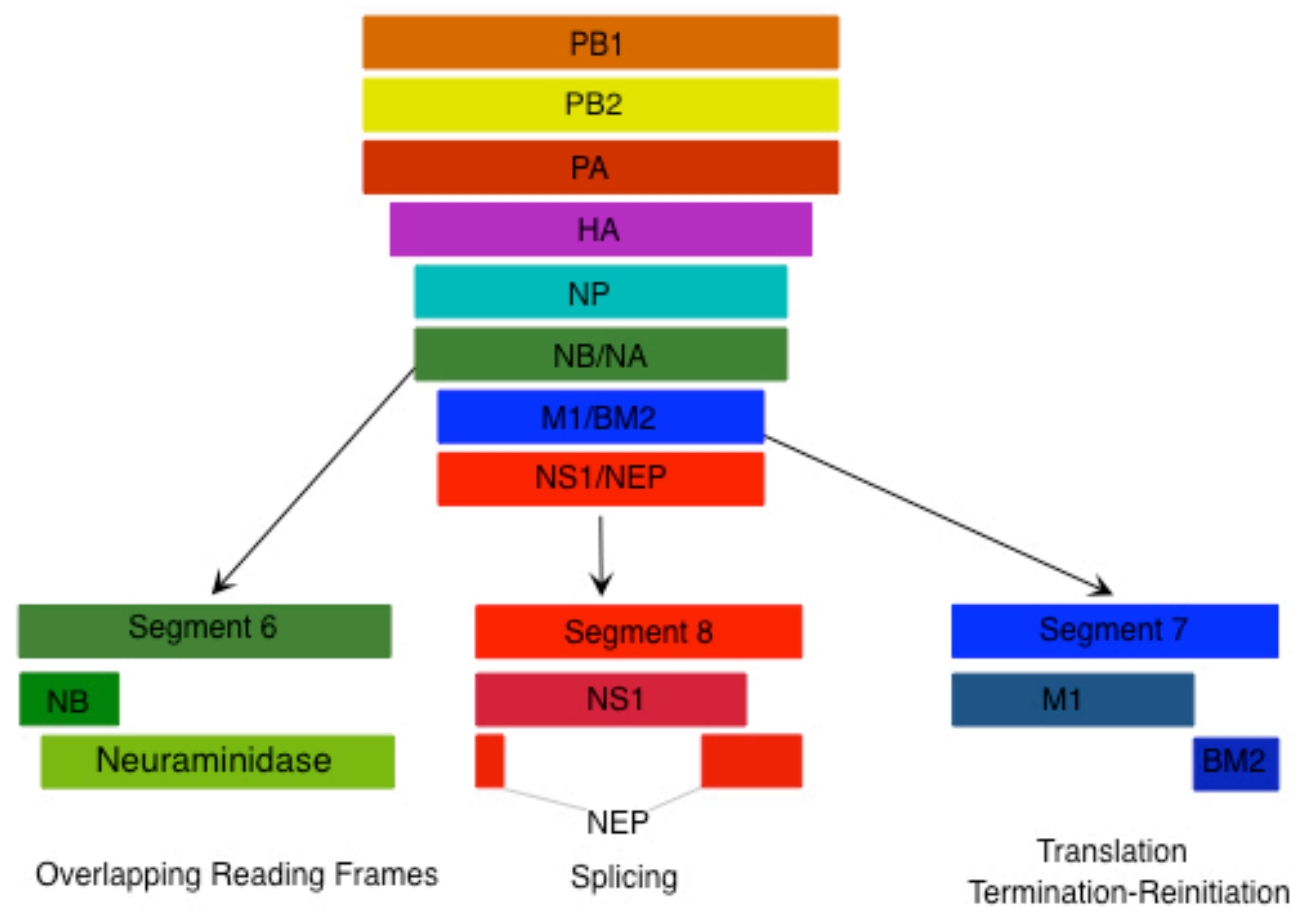

C)
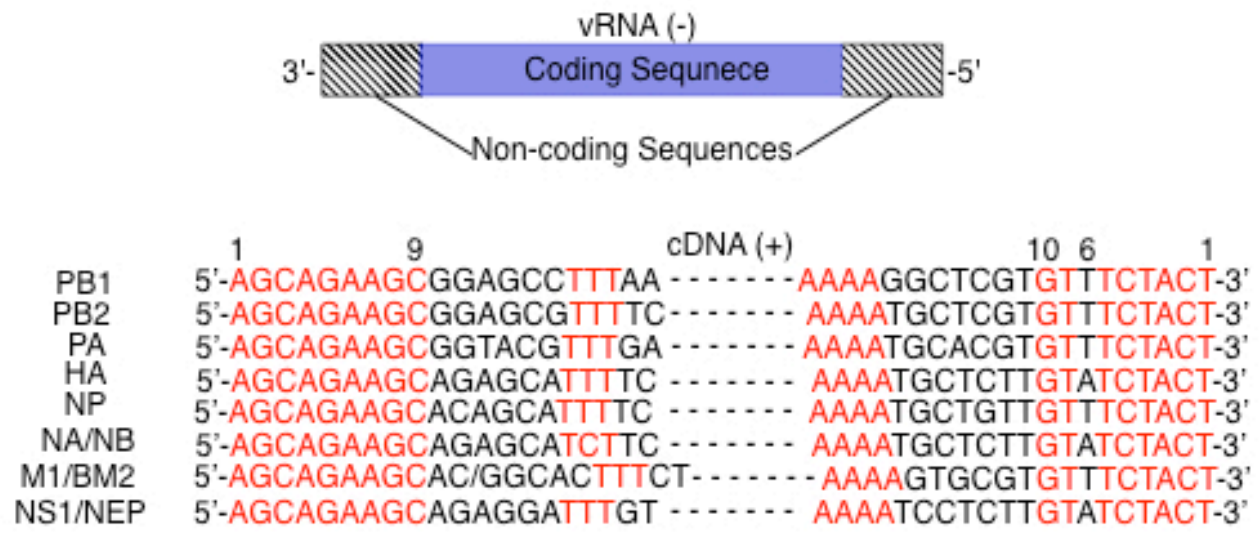

Sequences conserved among segments Segment-specific sequences

Figure 1 FluB Virus and Genome Organization 
Northern Hemisphere

\begin{tabular}{llll}
\hline Flu Season & Strain & Lineage of TIV strain & To be included in QIV \\
\hline $1998-1999$ & B/Beijing/184/93 & Yamagata & N/A \\
$1999-2000$ & B/Beijing/184/93 or B/Shangdong/7/97 & Yamagata & N/A \\
$2000-2001$ & B/Beijing/184/93 & Yamagata & N/A \\
$2001-2002$ & B/Sichuan/379/99 & Victoria & N/A \\
$2002-2003$ & B/Hong Kong/330/2001 & Victoria & N/A \\
$2003-2004$ & B/Hong Kong/330/2001 & Victoria & N/A \\
$2004-2005$ & B/Shanghai/361/2002 & Yamagata & N/A \\
$2005-2006$ & B/Shanghai/361/2002 & Yamagata & N/A \\
$2006-2007$ & B/Malaysia/2506/2004 & Victoria & N/A \\
$2007-2008$ & B/Malaysia/2506/2004 & Victoria & N/A \\
$2008-2009$ & B/Florida/4/2006 & Yamagata & N/A \\
$2009-2010$ & B/Brisbane/60/2008 & Victoria & N/A \\
$2010-2011$ & B/Brisbane/60/2008 & Victoria & N/A \\
$2011-2012$ & B/Brisbane/60/2008 & Victoria & N/A \\
$2012-2013$ & B/Wisconsin/1/2010 & Yamagata & B/Brisbane/60/2008 \\
$2013-2014$ & B/Massachusetts/2/2012 & Yamagata & B/Brisbane/60/2008 \\
$2014-2015$ & B/Massachusetts/2/2012 & Yamagata & B/Brisbane/60/2008 \\
$2015-2016$ & B/Phuket/3073/2013 & Yamagata & B/Brisbane/60/2008 \\
\hline
\end{tabular}

Southern Hemisphere

\begin{tabular}{llll}
\hline Flu Season & Strain & Lineage of TIV strain & To be included in QIV \\
\hline 1999 & B/Beijing/184/93 & Yamagata & N/A \\
2000 & B/Beijing/184/93 or B/Shangdong/7/97 & Yamagata & N/A \\
2001 & B/Sichuan/379/99 & Victoria & N/A \\
2002 & B/Sichuan/379/99 & Victoria & N/A \\
2003 & B/Hong Kong/330/2001 & Victoria & N/A \\
2004 & B/Hong Kong/330/2001 & Victoria & N/A \\
2005 & B/Shanghai/361/2002 & Yamagata & N/A \\
2006 & B/Malaysia/2506/2004 & Victoria & N/A \\
2007 & B/Malaysia/2506/2004 & Victoria & N/A \\
2008 & B/Florida/4/2006 & Yamagata & N/A \\
2009 & B/Florida/4/2006 & Yamagata & N/A \\
2010 & B/Brisbane/60/2008 & Victoria & N/A \\
2011 & B/Brisbane/60/2008 & Victoria & N/A \\
2012 & B/Brisbane/60/2008 & Victoria & N/A \\
2013 & B/Wisconsin/1/2010 & Yamagata & B/Brisbane/60/2008 \\
2014 & B/Massachusetts/2/2012 & Yamagata & B/Brisbane/60/2008 \\
2015 & B/Phuket/3073/2013 & Yamagata & B/Brisbane/60/2008 \\
\hline
\end{tabular}

Table 1 FluB Vaccines Strains in Northern and Southern Hemispheres 


\section{FluA}

FluB

\section{Phylogenetics}

- Subtyped by HA and NA (e.g. H1N1, H3N2)

- Two lineages according to HA: Victoria and Yamagata

\section{Epidemiology}

- More severe in children and elderly

\section{○}

\section{Clinical Presentation}

- Symptoms: soar throat, coughing, fever, nasal discharge

- Avian strains lead to severe disease and high mortality rates

\section{Virology}

- 8 segments, >13 known proteins

- Unique proteins: eg. PB1-F2, PB1-N40, PA-X

\section{Immunology}

- Generally, strong IFN $\alpha \beta$ and cytokine response

- HA is the major antibody target

- $\mathrm{M} 1$ and NP are the major CD8 ${ }^{+}$ $T$ cell antigens

- Two strains included in annual vaccine (one H3N2 and one H1N1)

\section{Antivirals}

- Adamantanes used are effective

- NAls effective, but resistance detected

\section{Host Range}

- Multiple animal reservoirs and possible animal hosts

- 8 segments, 11 known proteins

- Unique proteins: NB

- Strong IFN $\alpha \beta$ and cytokine response, details unclear

- $\mathrm{HA}$ is the major antibody target

- Major $\mathrm{CD}^{+} \mathrm{T}$ cell antigens are unknown

- One or two strains in annual vaccine depending on formulation

- Adamantanes are ineffective

- NAls effective, but resistance detected, in some cases without apparent fitness cost

- No established animal reservoirs 